\title{
A Numerical Algorithm for Solving Advection-Diffusion Equation with Constant and Variable Coefficients
}

\author{
S.G. Ahmed \\ Department of Engineering Physics and Mathematics, Faculty of Engineering, Zagazig Uninversity, P.B. Box 44519, \\ Zagazig, Egypt
}

\begin{abstract}
Abstarct: Advection-diffusion equation with constant and variable coefficients has a wide range of practical and industrial applications. Due to the importance of advection-diffusion equation the present paper, solves and analyzes these problems using a new finite difference equation as well as a numerical scheme. The developed scheme is based on a mathematical combination between Siemieniuch and Gradwell approximation for time and Dehghan's approximation for spatial variable. In the proposed scheme a special discretization for the spatial variable is made in such away that when applying the finite difference equation at any time level $(j+1)$ two nodes from both ends of the domain are left. After that the unknowns at the two nodes adjacent to the boundaries are obtained from the interpolation technique. The results are compared with some available analytical solutions and show a good agreement.
\end{abstract}

Keywords: Advection-diffusion equation, Explicit finite difference techniques, Implicit finite difference techniques, Interapolation techniques.

\section{INTRODUCTION}

Advection-diffusion equation is one of the most important partial differential equations and observed in a wide range of engineering and industrial applications [1]. It has been used to decsribe heat transfer in a draining film [2], water transfer in soil [3], dispersion of tracers in porous media [4], contaminant dispersion in shallow lakes [5], the spread of solute in a liquid flowing through a tube, longrange transport of pollutants in the atmosphere [6] and dispersion of dissolved salts in groundwater [7]. In the initial works while obtaining the analytical solutions of dispersion problems in the ideal conditions, the basic approach was to reduce the advection-diffusion equation into a diffusion equation by eliminating the advection term(s). It was done either by introducing moving coordinates see, Ogata Banks 1969 [8]; Harleman and Rumer 1963 [9]; Bear 1972 [10]; Guvanasen and Volker 1983 [11]; Aral and Liao 1972 [12] and Marshal et al., 1996 [13]. Another direction is to transform advection-diffusion to diffusion equation only was by introducing another dependent variable see Banks and Ali 1964 [14]; Ogata 1970 [15]; Lai and Jurinak 1971 [16]; Marin 1974 [17] and Al-Niami and Rushton 1977 [18]. Some one-dimensional analytical solutions have been given, see Tracy 1995 [19] by transforming the nonlinear advection-diffusion into linear one for specific forms of the moisture contents $v s$ pressure head and relative hydraulic conductivity $v s$ pressure head curves which allow both twodimensional and three-dimensional solutions to derived. Accurate numerical solution of the advection-diffusion

*Address correspondence to this author at the Department of Engineering Physics and Mathematics, Faculty of Engineering, Zagazig Uninversity, P.B. Box 44519, Zagazig, Egypt; Tel: +20106392877;

E-mail:sgahmed911@hotmail.com equation is usually characterized by a dimensionless parameter, called Peclect number. These results become increasingly difficult as the Peclect number increases due to onset of spurious oscillations or excessive numerical damping if finite difference [20] or finite element formulations are used [21]. Numerous innovative algorithms and methods can be found in the literatures [22-31]. In finding the analytical solutions many difficulties encountered such as the nonlinearities etc. also in the numerical solutions difficulties apear due to many reseaons and herein we will give two for such reseaons. Firstly, the nature of the governing equation, which includes first-order and secondorder partial derivatives in space. Secondly, it is vital to construct an appropriate mesh to obtain a better approximation to the problem. However, the construction of an appropiate mesh is not easy task and sometimes the problem can not be solved because the lake of mesh structure. In the present paper, the advection-diffusion equation with constant and variable coefficients is solved using a new scheme. The developed scheme is based on a mathematical combination between Siemieniuch and Gradwell approximation for time and Dehghan's approximation for spatial variable. The scheme also developed and based on a special discretization for spatial variable in such away that when applying the finite difference equation at any time level $(j+1)$ two nodes from both ends of the domain are left and by assuming all unknowns became known at the old time level $(j)$, the new finite difference equation is applied at $m-2$ internal nodes of the domain, where $m$ are the total number of nodes inside the domain of interest. Then making use of interpolation technique to find the unknows at two internal nodes left. After that the unknowns at the two nodes adjacent to the boundaries are obtained from the interpolation technique. Four examples with known analytical solutions are presented 
to test the validity of the new finite difference equation and its new scheme. The first three examples are of constant coefficients and are chosen such that different cases between the advection and diffusion parameters are used to show their affect on the solution. The fourth example is for advection-diffusion equation with variable coefficients. It was for one-dimensional advection-diffusion equation in a longitudal finite initially solute free domain and for dispersion problem. In this example the velocity of the flow considered spatially dependent due to inhomogenity of the domain. Results due to the present method agree well with the analytical solutions and can be extend to cover higher dimensional problems.

\section{MATHEMATICAL FORMULATION}

In the present paper, the mathematical formulation for two different types of advection-diffusion equation will be presented. The first type is for advection-diffusion equation with constant coefficients while the second type is for the same equation but with variable coefficients.

Case (1): Advection-diffusion with constant coefficients:

$\frac{\partial u}{\partial t}+\beta \frac{\partial u}{\partial x}=\alpha \frac{\partial^{2} u}{\partial x^{2}}$

With initial condition

$u(x, 0)=f(x) \quad 0 \leq x \leq \ell$

And boundary conditions

$u(x=0, t)=g_{0}(x) \quad 0<t \leq T$

$u(x=\ell, t)=g_{1}(x) \quad 0<t \leq T$

Condition (3) or (4) may be replaced by:

$\frac{\partial u(x, t)}{\partial x}=g_{2}(x) \quad 0<t \leq T$

In equations (2-5) the functions $f(x), g_{0}(x), g_{1}(x)$ and $g_{2}(x)$ are known.

Case (2): Advection-diffusion with variable coefficients:

As we mentioned before, advection-diffusion equation can be found in different types of practical applications, and so the variable coefficients will have different meaning from application to another. Therefore, the mathematical formulation for advection-diffusion herein, will be for solute dispersion problem. In this problem, the medium is considered inhomogeneous, therefore the velocity is considered depend on the position variable. The mathematical formulation for such application will be as follow:

$\frac{\partial C(x, t)}{\partial t}=\frac{\partial}{\partial x}\left(D(x, t) \frac{\partial C(x, t)}{\partial x}-u(x, t) C(x, t)\right)$

With

$u(x, t)=u_{0}(1+a x)$
$D(x, t)=D_{0}(1+a x)^{2}$

$C(x, t)=0,0 \leq x \leq \ell, t=0$

$C(x, t)=C_{0}, x=0, t>0$

$\frac{\partial C(x, t)}{\partial x}=0, x=\ell, t \geq 0$

In fact the mathematical formulation in both cases is the same, only two equations are added in the second case and given by equations (7) and (8). This difference comes from the nature of the dispersion problem that is both flow velocity and dispersion should satisfy Darcy's law, therefore, Atul in [30] added these two relations to ensure satisfication of Darcy's law.

\section{THE PROPOSED FINITE DIFFERENCE EQUATION}

The scheme starts by using the Siemieniuch-Gradwell time difference formula as follow:

$$
\begin{aligned}
\left(\frac{\partial u}{\partial t}\right)_{i, n}= & A_{11}\left(u_{i-1, n+1}-u_{i-1, n}\right)+B_{11}\left(u_{i, n+1}-u_{i, n}\right)+ \\
& C_{11}\left(u_{i+1, n+1}-u_{i+1, n}\right)
\end{aligned}
$$

Next use the space difference formulas derived by Dehghan [29]:

$$
\begin{aligned}
\left(\frac{\partial u}{\partial x}\right)_{i, n}= & A_{21}\left(u_{i+2, n}-u_{i, n}\right)+B_{21}\left(u_{i, n}-u_{i-2, n}\right)+ \\
& C_{21}\left(u_{i+1, n}-u_{i-1, n}\right)
\end{aligned}
$$

And

$$
\begin{aligned}
\left(\frac{\partial^{2} u}{\partial x^{2}}\right)_{i, n}= & A_{31}\left(u_{i+1, n}-2 u_{i, n}+u_{i-1, n}\right)+ \\
& B_{31}\left(u_{i+2, n}-2 u_{i, n}+u_{i-2, n}\right)+C_{21}\left(u_{i+1, n}-u_{i-1, n}\right)
\end{aligned}
$$

Substutiting equations (12-14) into equation (1) and rearrange according the time level, lead to:

$$
\begin{aligned}
& \left(A_{11} u_{i-1}+B_{11} u_{i}+C_{11} u_{i+1}\right)^{n+1}= \\
& \left(\xi_{1} u_{i-2}+\xi_{2} u_{i-1}+\xi_{3} u_{i}+\xi_{4} u_{i+1}+\xi_{5} u_{i+2}\right)^{n}
\end{aligned}
$$

In which:

$$
\begin{aligned}
& \xi_{1}=\beta B_{21}+\alpha B_{31} \\
& \xi_{2}=A_{11}+\beta C_{21}+\alpha A_{31} \\
& \xi_{3}=B_{11}+\beta A_{21}-\beta B_{21}-2 \alpha A_{31}-2 \alpha B_{31} \\
& \xi_{4}=C_{11}-\beta C_{21}+\alpha A_{31} \\
& \xi_{5}=-\beta A_{21}+\alpha A_{31} \\
& A_{11}=C_{11}=\frac{2\left[\beta\left(\frac{\Delta t}{(\Delta x)^{2}}\right)\right]-\left[\beta\left(\frac{\Delta t}{\Delta x}\right)\right]}{4(\Delta t)}
\end{aligned}
$$


$B_{11}=\frac{2+\left[\beta\left(\frac{\Delta t}{\Delta x}\right)\right]-2\left[\beta\left(\frac{\Delta t}{(\Delta x)^{2}}\right)\right]}{2(\Delta t)}$

$A_{21}=\frac{12\left[\beta\left(\frac{\Delta t}{(\Delta x)^{2}}\right)\right]+2\left[\beta\left(\frac{\Delta t}{\Delta x}\right)\right]^{2}-3\left[\beta\left(\frac{\Delta t}{\Delta x}\right)\right]-2}{24(\Delta x)}$

$A_{31}=\frac{-\left[\beta\left(\frac{\Delta t}{\Delta x}\right)\right]^{4}-8\left[\beta\left(\frac{\Delta t}{\Delta x}\right)\right]^{2}-12\left[\beta\left(\frac{\Delta t}{(\Delta x)^{2}}\right)\right]^{2}+8\left[\beta\left(\frac{\Delta t}{(\Delta x)^{2}}\right)\right]}{6\left[\beta\left(\frac{\Delta t}{(\Delta x)^{2}}\right)\right](\Delta x)^{2}}$

$B_{21}=\frac{2\left[\beta\left(\frac{\Delta t}{\Delta x}\right)\right]^{2}+12\left[\beta\left(\frac{\Delta t}{(\Delta x)^{2}}\right)\right]+3\left[\beta\left(\frac{\Delta t}{\Delta x}\right)\right]-2}{24(\Delta x)}$

$B_{31}=\frac{\left[\beta\left(\frac{\Delta t}{\Delta x}\right)\right]^{4}-8\left[\beta\left(\frac{\Delta t}{\Delta x}\right)\right]^{2}+12\left[\beta\left(\frac{\Delta t}{(\Delta x)^{2}}\right)\right]^{2}-2\left[\beta\left(\frac{\Delta t}{(\Delta x)^{2}}\right)\right]}{6\left[\beta\left(\frac{\Delta t}{(\Delta x)^{2}}\right)\right](\Delta x)^{2}}$

$C_{21}=(-) \frac{\left[\beta\left(\frac{\Delta t}{\Delta x}\right)\right]^{2}+6\left[\beta\left(\frac{\Delta t}{(\Delta x)^{2}}\right)\right]-4}{6(\Delta x)}$

The $(m-2)$ linear system of equations obtained from applying equation $(15)$ at the time levels $(j+1)$ and $(j)$, respectively.

\section{SOLUTION ALGORITHM}

Suppose that the domain of the problem is divided into $m$ internal points, see Fig. (1), then at each two successive times level apply equation (15), leads to a linear system of equations.

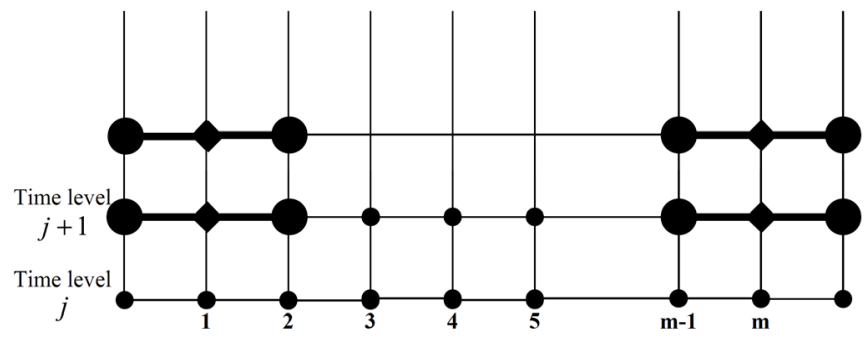

Fig. (1). Grid distribution.

The new scheme can be stated as follows:

1. Suppose that the domain $0<x<\ell$ is divided into $m$ internal nodes and numbered according to Fig. (1).

2. At time level $(j)$ all temperature nodes should be known from previous time level and this represents the R.H.S. of equation (15).
3. At time level $(j+1)$ apply the L.H.S. of equation (15) starting from node (2) and stop at node $(m-1)$.

4. Solve the linear system of equation resulted from steps 3 and 4 .

5. Use interpolation technique to find the temperature at the internal nodes 1 and $(m)$.

6. Repeat steps (2-5) up to the end of the time.

\section{RESULTS AND DISCUSSION}

The proposed finite difference equation and the numerical scheme are tested for their validity to solve advection-diffusion with constant and variable coefficients. Three different examples for advection-diffusion with constant coefficients are solved to study the effect of the parameters $\alpha$ and $\beta$ on the results obtained, then another two different examples the first one is a one-dimensional advection-diffusion equation with variable coefficients and the second one is a two-dimensional case problem. Let us start with the first three examples of constant coefficients to study the effect of the parameters $\alpha$ and $\beta$

Problem (1): 1-D Advection-Diffusion with $\alpha=0.01 \& \beta=1.0$

In this case problem, the constant coefficients appear in the advection-diffusion equation are $\alpha=0.01 \& \beta=1.0$ associated with initial and boundary conditions respectively, given by:

$u(x, 0)=f(x)=\exp \left(-\frac{(x+0.5)^{2}}{0.00125}\right)$

$u(x=0, t)=g_{0}(x)=\frac{0.025}{\sqrt{0.000625+0.02 t}} \exp \left(-\frac{(0.5-t)^{2}}{0.00125+0.04 t}\right)$

$u(x=1, t)=g_{1}(x)=\frac{0.025}{\sqrt{0.000625+0.02 t}} \exp \left(-\frac{(1.5-t)^{2}}{0.00125+0.04 t}\right)$

The exact solution is given by:

$u(x, t)=\frac{0.025}{\sqrt{0.000625+0.02 t}} \exp \left(-\frac{(x+0.5-t)^{2}}{0.00125+0.04 t}\right)$

The problem is solved at different cases for space size step and time size step. In Fig. (2), the results for two different space size step and two different time step size step are shown. It is very clear that a good agreement between the analytical solution and the present numerical results with minimum error obtained, and the error becomes clear when using large size step for time and space.

Problem (2): 1-D Advection-Diffusion with $\alpha=1.0 \& \beta=0.1$

In this case problem, the constant coefficients appear in the advection-diffusion equation are $\alpha=1.0 \& \beta=0.1$ associated with initial and boundary conditions respectively, given by: 


$$
\begin{aligned}
& u(x, t)=0.0 \\
& u(x=0, t)=g_{0}(x)=300 \quad 0<t \leq T \\
& \frac{\partial u(x=6, t)}{\partial x}=g_{1}(x) \quad 0<t \leq T
\end{aligned}
$$

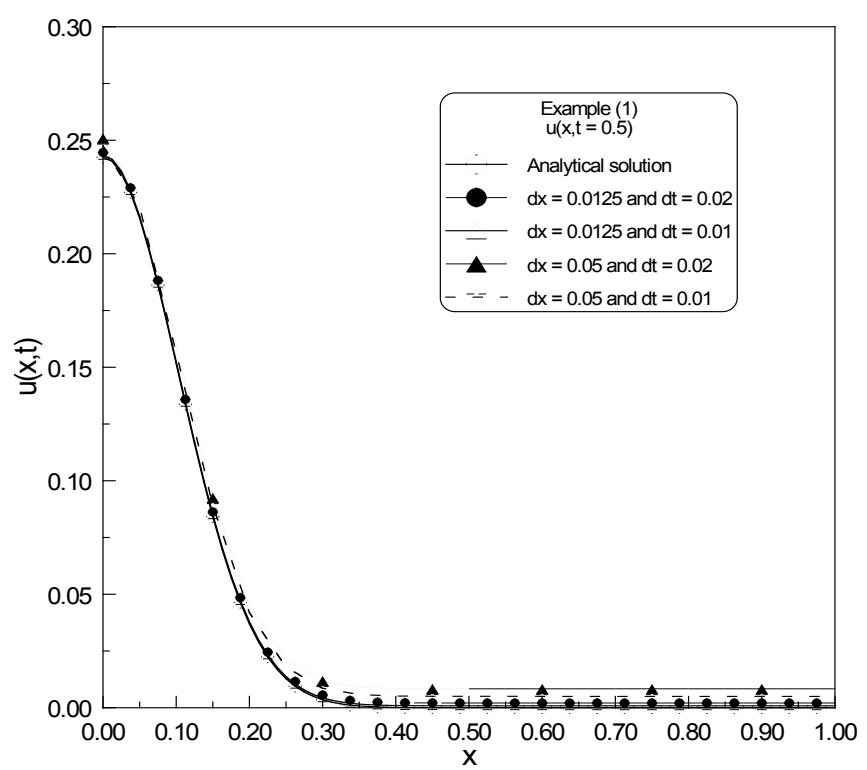

Fig. (2). Results of example (1).

In this case, the example is solved at three different time steps; $0.1,1$ and 3 . The results are compared with the corresponding analytical solution as shown in Fig. (3). As it appears from the figure, a good agreement between the analytical solutions and the present results can be observed.

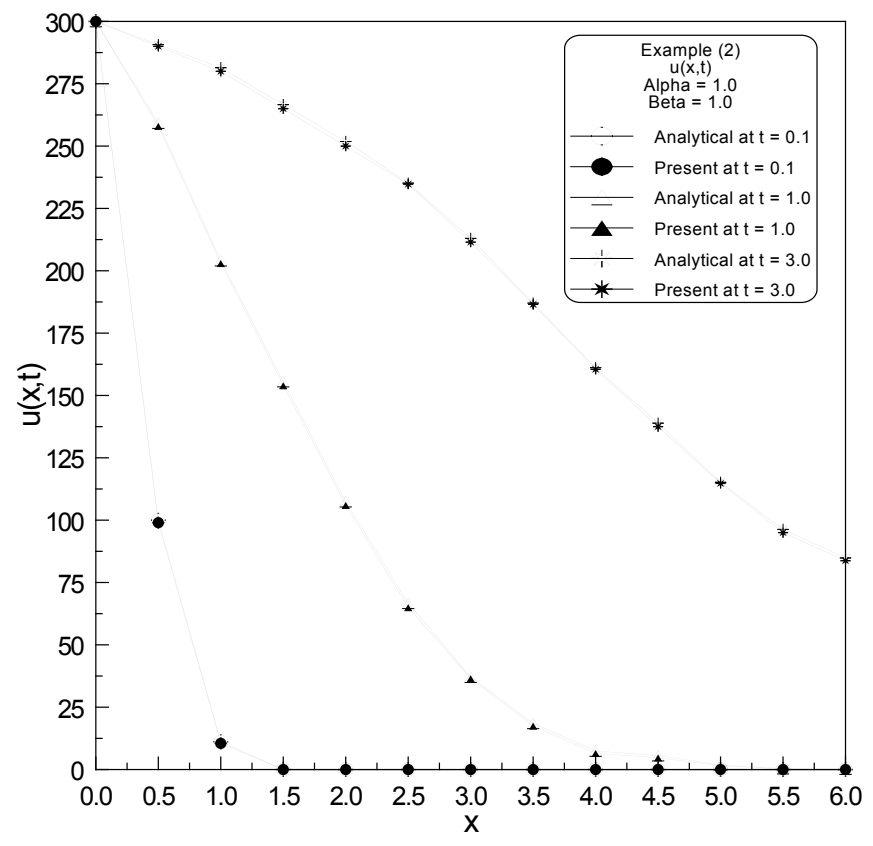

Fig. (3). Results of example (2).

$$
\begin{gathered}
\text { Problem (3): } \quad 1-\mathrm{D} \quad \text { Advetion-Diffusion with } \\
\alpha=1.0 \& \beta=2.0
\end{gathered}
$$

In this case problem, the constant coefficients appear in the advection-diffusion equation are $\alpha=1.0 \& \beta=2.0$ associated with initial and boundary conditions respectively, given by:

$u(x, t)=0.0$

$u(x=0, t)=a \exp (b t) \quad 0<t \leq T$

$u(x=6, t)=a \exp (b t+c x) \quad 0<t \leq T$

$u(x, t=0)=a \exp (c x)$

The analytical solution of this problem is given by:

$u(x, t)=a \exp (b t+c x), c=\frac{\alpha \pm \sqrt{\alpha^{2}+4 \beta b}}{2 \beta}$

The temperature variation against spatial variable and at different times are shown in Fig. (4). It is clear from the figure. that a linear damping is occurred and the error becomes large by inreasing the space and becomes clear at large times, but in all cases the error is acceptable and can be decreased by decreasing both space and time step size.



Fig. (4). Results of example (3).

Problem (4): 1-D Advection-Diffusion with variable coefficients

The fourth case problem is for the same equation with variable coefficients. The solute dispersion problem is taken as a practical example. The domain of the problem $0 \leq x \leq 1.0(\mathrm{Km})$ is inhomogeneous, therefore the velocity is considered dependent on the position variable. An important parameter called inhomogeneous parameter is taken into consideration and its effect on the results studied for two different values away from each others as shown in Figs. (57). Starting the results by Fig. (5) which shows the variation of the ratio $C / C_{0}, C_{0}=1$ against the spatial variable $x$ at different times $0.1 \leq t \leq 1.0$ and at inhomogeneous parameter equals $a=1.0$. As it is clear that the behavior in all curves is exponentially decaying and by going the time up the ratio increases at any spatial point. Following up the 
results and turn up to Fig. (6) which shows the effect of the inhomogeneous parameter on similar results obtained in Fig. (5) but in this case the inhomogeneous parameter equals $a=0.1$. Obviously the same behavior is obtained but the exponently decaying behavior becomes more concave downward, i.e., at the same point $x$ the ratio $C / C_{0}$ in case of $a=1.0$ is greater when $a=0.1$ and agrees very well with the practice. It is clear from both Figs. $(\mathbf{5}, \mathbf{6})$ that by increasing the inhomogeneous parameter, the ratio $C / C_{0}$ increases and vise versa. Collecting these two figures in one figure. to clarify this observation which becomes very clear as shown in Fig. (7). Finally, from the solution of the above four test problems one can see that the proposed equation and scheme are simple in computation compared with other methods.

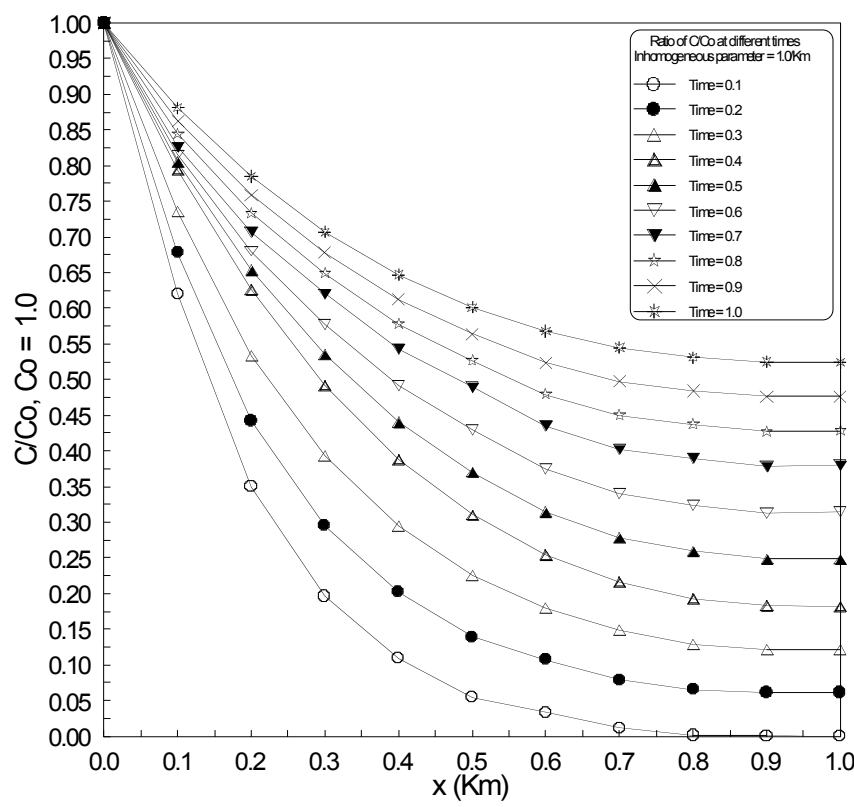

Fig. (5). Ratio of $C / C_{0}$ at different times and inhomogeneous parameter equals 1.0 .

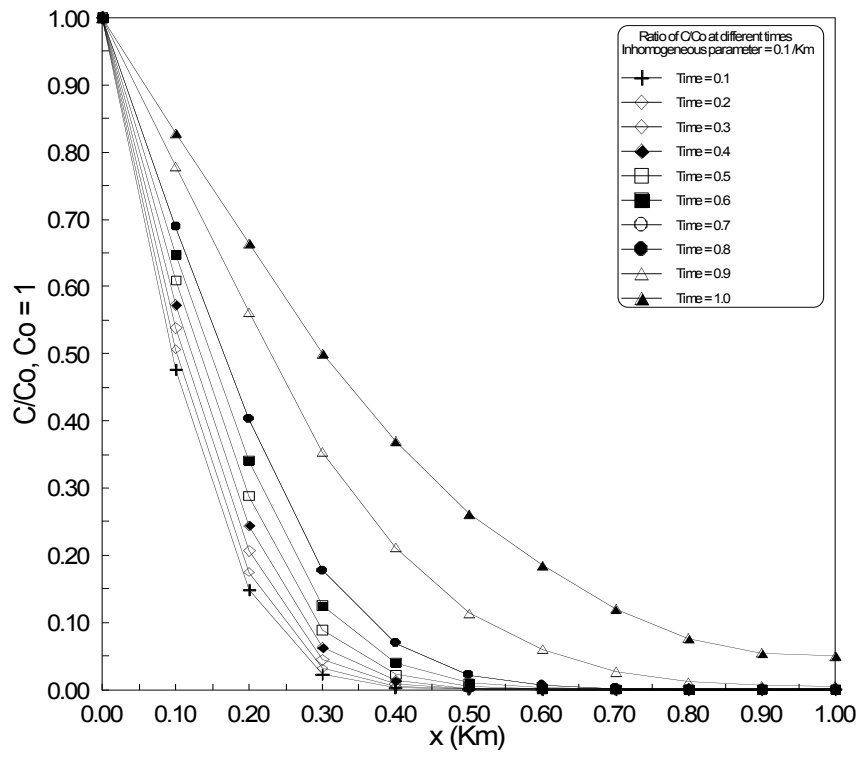

Fig. (6). Ratio of $C / C_{0}$ at different times and inhomogeneous parameter equals 0.1 .

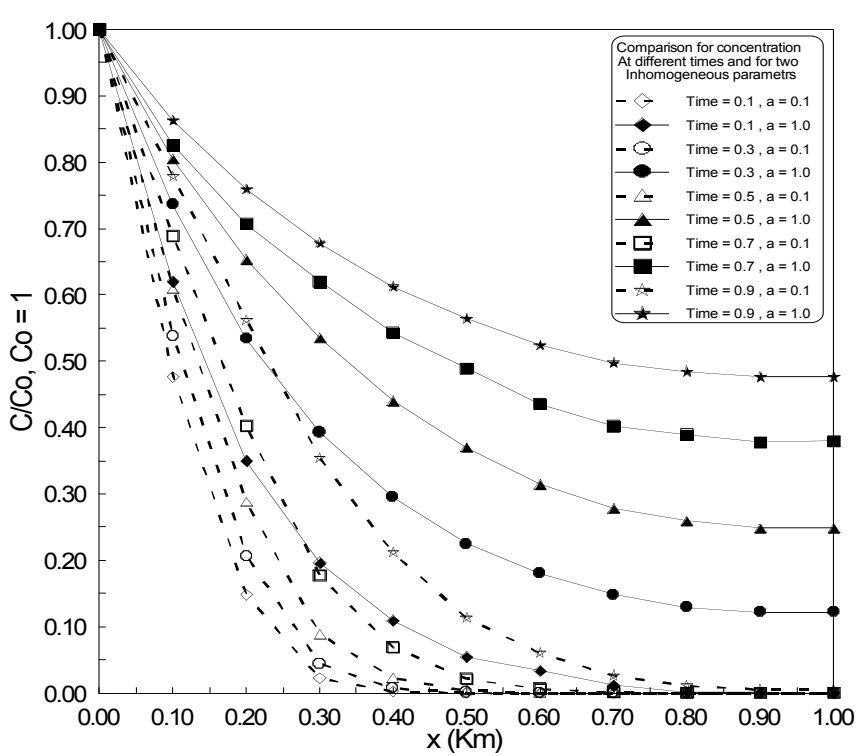

Fig. (7). Comparison between $C / C_{0}$ at different times and two diferent values for inhomogeneous parameter.

Problem (5): 2-D Advection-Diffusion with variable coefficients

In this test problem, a two-dimensional advectiondiffusion equation with variable coefficients are solved and the obtained results are compared with the available analytical solution. The government differential equation is:

$$
\begin{aligned}
\frac{\partial u(x, y, t)}{\partial t}= & k_{x} \frac{\partial^{2} u(x, y, t)}{\partial x^{2}}+k_{y} \frac{\partial^{2} u(x, y, t)}{\partial y^{2}} \\
& +v_{x} \frac{\partial u(x, y, t)}{\partial x}+v_{y} \frac{\partial u(x, y, t)}{\partial y}
\end{aligned}
$$

$0 \leq \mathrm{x}, \mathrm{y} \leq 1$

The exact solution is given by;

$u^{e x}(x, y, t)=a\left(\exp \left(b t+c_{x} x\right)+\exp \left(b t+c_{y} y\right)\right)$

$\mathrm{c}_{\mathrm{x}}=\frac{v_{x} \pm \sqrt{v_{x}^{2}+4} k_{x} b}{2 k_{x}}$

$\mathrm{c}_{\mathrm{y}}=\frac{v_{y} \pm \sqrt{v_{y}^{2}+} 4 k_{y} b}{2 k_{y}}$

The initial and boundary conditions are obtained from the analytic solution as follows:

$$
\begin{aligned}
& u(x, y, 0)=u^{e x}(x, y, 0) \\
& u(0, y, t)=u^{e x}(0, y, t) \quad, u(1, y, t)=u^{e x}(1, y, t) \\
& u(x, 0, t)=u^{e x}(x, 0, t) \quad, u(x, 1, t)=u^{e x}(x, 1, t)
\end{aligned}
$$

The results for this test problem are shown in Figs. (813). In these figures a comparison between the results due to the present results and the analytical solution at three different times, $t=0.1,0.5,0.9$ seconds. As it is clear from these figures, a good agreement between the two results are obtained. 


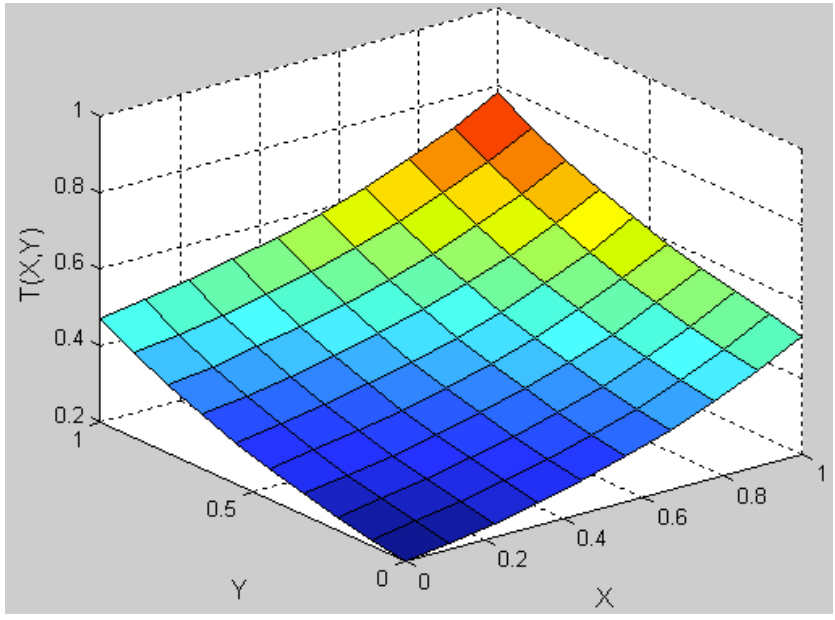

Fig. (8). Analytical solution at $\mathrm{t}=0.1$.

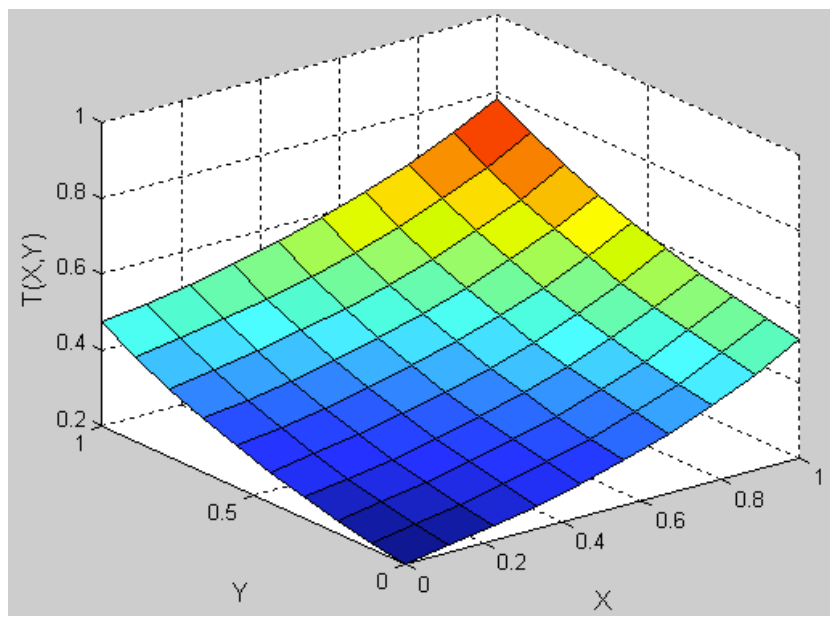

Fig. (9). Present at $t=0.1$.

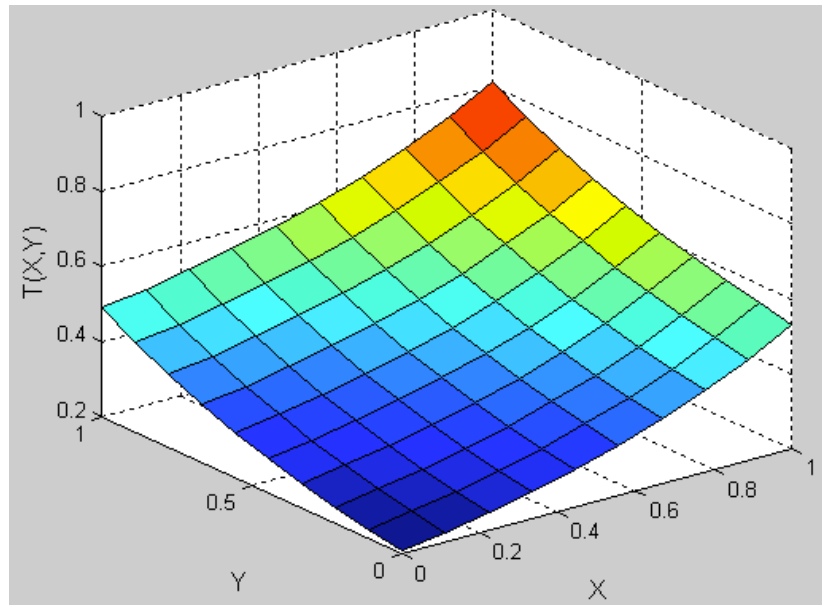

Fig. (10). Analytical at $\mathrm{t}=0.5$.

\section{CONCLUSION}

In the present paper, a new finite difference equation is derived to solve linear advection-diffusion equation with both constant and variable coefficients. The derivation based on a mathematical combination between spatial and time discretisation from previous numerical methods. Also a new numerical scheme is developed to track the unknowns in the problem under condideration. Due to the practical importance of the advection-diffusion equation, the present method can open the way to the reseachers to this direction of method of computation due to easy manuplation and the high accuracy obtained as seen from the five test examples in one and two dimensions that solved in the present paper. Finally one can modify the present method by using different finite difference methods for approximating both spatial and time derivatives.

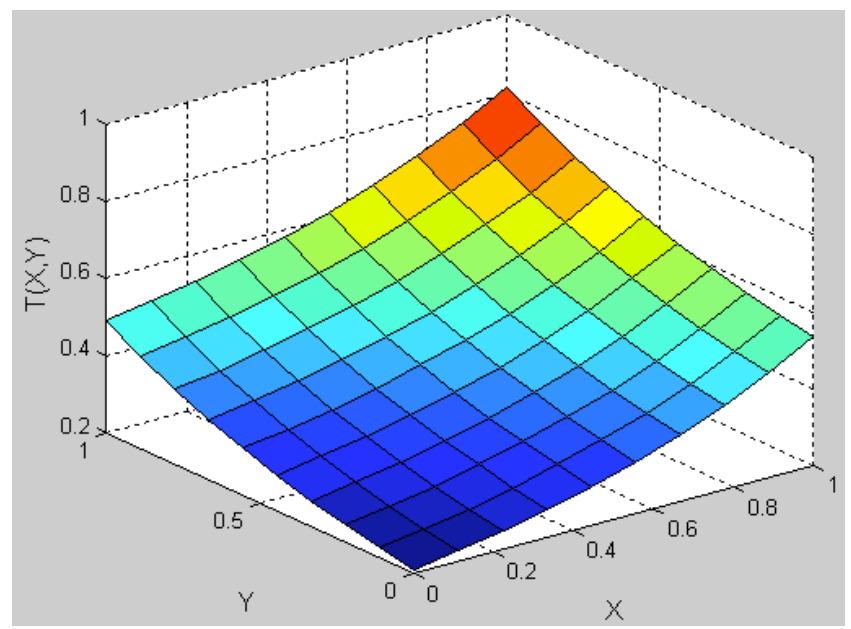

Fig. (11). Present at $t=0.5$.

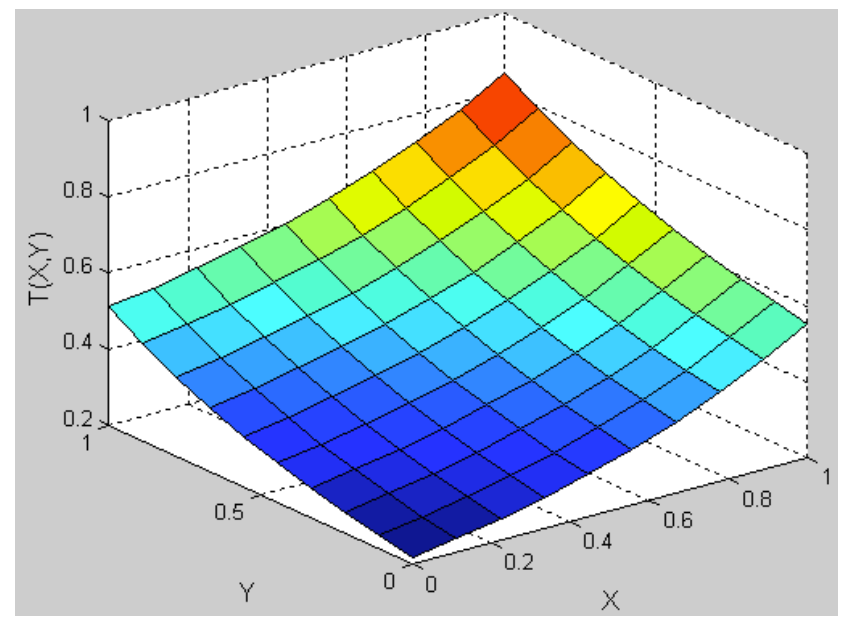

Fig. (12). Analytical at $\mathrm{t}=0.9$.

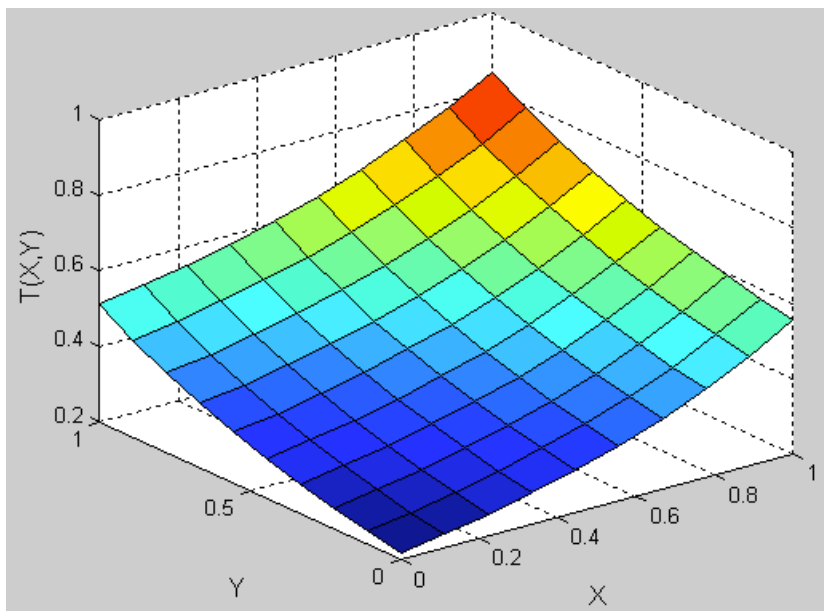

Fig. (13). Present at $t=0.9$. 


\section{NOMENCLATURE}

$C(x, t)=$ Solute concentration at position $x$ along the longitudal direction at a time $t$

$D(x, t)=$ Solute dispersion

$u(x, t) \quad=$ Medium's flow velocity

$a \quad=$ Inhomogeneous parameter, $a=\frac{b}{\ell}$

$b \quad=$ May be real constant

\section{ACKNOWLEDGEMENTS}

None declared.

\section{CONFLICT OF INTEREST}

None declared.

\section{REFERENCES}

[1] Singh KM, Tanaka M. On exponential variable transformation based boundary element formulation for advection-diffusion problems. Eng Anal Bound Elem 2000; 24: 225-35.

[2] Isenberg J, Gutfinger C. Heat transfer to a draining film. Int J Heat Transfer 1972; 16: 505-12.

[3] Parlarge JY. Water transport in soils. Ann Rev Fluids Mech 1980; 2: $77-102$

[4] Fattah QN, Hoopes JA. Dispersion in anistropic homogenoeous porous media. J Hydraul Eng 1985; 111: 810-27.

[5] Salmon JR, Ligett JA, Gallager RH. Dispersion analysis in homogeneous lakes. Int J Numer Meth Eng 1980; 15: 1627-42.

[6] Zlatev Z, Berkowicz R, Prahm LP. Implementation of a variable stepsize variable formula in the time-integration part of a code for treatment of long-range transport of air pollutants. J Comp Phys 1984; 55: 278-301.

[7] Guvanasen V, Volker RE. Numerical solution for solute transport in unconfined aquifers. Int J Numer Meth Fluids 1983; 3: 103-23.

[8] Ogata A, Banks RB. A solution of the differential equation of longitudinal dispersion in porous media. U.S. Geol Surv Prof Paper 1961; 411-A: 1-9.

[9] Harleman DRF, Rumer RE. Longitudinal and lateral dispersion in an isotropic porous medium. J Fluid Mech 1963; 385-94.

[10] Bear J. Dynamics of fluids in porous media. New York: Am Elesiver Co. 1972; p. 764.

[11] Guvanasen V, Volker RE. Experimental investigations of unconfined aquifer pollution from recharge basins. Water Resour Res 1983; 19(3): 707-17.
[12] Aral MM, Liao B. Analytical solution for two-dimensional transport equation with time-dependent dispersion coefficients. J Hydrol Engg 1996; 1(1): 20-32.

[13] Marshal TJ, Holmes JW, Rose CW. Soil Physics. $3^{\text {rd }}$ ed. Cambridge: Cambridge University Press 1996.

[14] Banks RB, Ali J. Dispersion and adsorption in porous media flow. J Hydraul Div 1964; 90: 13-31.

[15] Ogata A. Theory of dispersion in granular media. US Geol Sur Prof Paper 1970; 411-1: 34

[16] Lai SH, Jurinak JJ. Numerical approximation of cation exchange in miscible displacement through soil columns. Soil Sci Soc Ami 1971; 35: 894-9.

[17] Al-Nami ANS, Rushton KR. Analysis of flow against dispersion in porous media. J Hydrol 1977; 33: 87-97.

[18] Spalding DB. A noval finite difference formulation for differential expressions involving both first and second derivatives. Int $\mathbf{J}$ Numer Meth Eng 1972; 4: 551-9.

[19] Hughes TJR, Brooks AA. A theoretical framwork for petrovGalerkin methods with discontinuous weighting functions: application to the streamline-upwind procedure. In: Gallagher RH, Norrie DH, Oden JT, Zienkiewicz OC, Eds. Finite Elements in Fluids 4, London: Wiley 1982; pp. 47-65.

[20] Donea J, Giulinai S, Laval H. Quartapelle, time accurate solution of advection-diffusion problems by finite elements. Comput Methods Appl Mech Eng 1984; 45: 123-45.

[21] Yu CC, Heinrich JC. Petrov-Galerkin methods for the timedependent convective transport equation. Int $\mathrm{J}$ Numer Meth Eng 1986; 23: 883-901.

[22] Westerink JJ, Shea D. Consistent higher higher degree PetrovGalerkin methods for solution of the transient convection-diffusion equation. Int J Numer Meth Eng 1989; 29: 1077-101.

[23] Park NS, Ligget JA. Taylor-least-squares finite element for twodimensional advection-dominated unsteady advection-diffusion problems. Int J Numer Meth In Fluids 1990; 11:21-38.

[24] Morton KW. Numerical solution of convection-diffusion equation. London: Chapan \& Hall 1996.

[25] Kansa EJ. Multiquadrics: a scattered data approximation scheme with application to computational fluid-dynamics. Comput Math Appl 1990; 19: 147.

[26] Thomas JW. Numerical partial differential equations: finite difference methods. Berlin, New York: Springer-Verlag 1995.

[27] Dehghan M. Weigthed finite difference techniques for the onedimensional advection-diffusion equation. Appl Math Comput 2004; 147: 307-19.

[28] Dehghan M. Quasi-implicit and two-level explicit finite-difference procedures for solving the one-dimensional advection equation. Appl Math Comput 2005; 167: 46-67.

[29] Dehghan M. Numerical solution of three-dimensional advectiondiffusion equation. Appl Math Comput 2004; 150: 5-19.

[30] Dehghan M. Time-spliting procedures for the solution of the twodimensional transport equation. Kybernetes 2007; 36: 791-805.

[31] Dehghan M. On the numerical solution of the one-dimensional convection-diffusion equation. Mathl Probl Eng 2005; 61-74. 